\title{
Direct atomistic simulations of metastable state destruction in titanium $(\beta-\alpha$ martensitic transition) caused by external influences
}

\author{
Pavel V. Chirkov ${ }^{1}$, Roman M. Kichigin ${ }^{1,2}$, Alexey V. Karavaev ${ }^{1,2^{*}}$ and Vladimir V. Dremov ${ }^{1}$ \\ ${ }^{1}$ Federal State Unitary Enterprise "Russian Federal Nuclear Center - Zababakhin All-Russia Research \\ Institute of Technical Physics", Snezhinsk, 454070 Russia \\ ${ }^{2}$ Snezhinsk Physics and Technology Institute, National Research Nuclear University "MEPhI" \\ (Moscow Engineering Physics Institute), Snezhinsk, 454070 Russia
}

\begin{abstract}
Large-scale classical molecular dynamics (CMD) is utilized to simulate the $\beta \rightarrow \alpha$ phase transition in pure titanium. Samples with a metastable polycrystalline $b c c$ structure are prepared using crystallization from liquid state and subsequent recrystallization at elevated temperatures. Controlling the heating-cooling regimes we prepared two different kinds of samples with coarse and fine grain structures. The metastable $b c c$ samples were relaxed at temperatures noticeably lower than the equilibrium $\beta-\alpha$ transition temperature. During the following cooling of the samples down to room temperature, transitions to the $\alpha$ phase start. With the prepared metastable $b c c$ samples of two kinds we perform the CMD study of the $\beta \rightarrow \alpha$ transition under plain shock wave loading and imposed shear deformations. From the CMD simulations we obtain information about the transformation barriers, mechanisms, and kinetics. Results of CMD simulations suggest that grain boundaries hamper the $h c p$ phase growth.
\end{abstract}

\section{Introduction}

Martensitic phase transitions have a great influence on the properties of such materials as steels, shape-memory alloys, and titanium based alloys. Due to a unique combination of low density and high strength Ti-based alloys are of a great technological importance as structural materials. There are found three crystal phases of pure Ti under pressures up to $80 \mathrm{GPa}$ [1]. At ambient conditions the $\alpha$ phase with a hexagonal closed-packed $(h c p)$ lattice is stable. At temperatures above $1155 \mathrm{~K}$ and $P=0$ the transition to the body-centered cubic (bcc) $\beta$ phase takes place. Under pressures higher than $\sim 5 \mathrm{GPa}$ the $\alpha$ phase transforms into the simple hexagonal $\omega$ phase. In this work we focus on the investigation of the $h c p \leftrightarrow b c c$ transition under external pressures and shear deformations, which is the basis of titanium alloys technological processes like cooling during solidification, heat and mechanical treatments. The fundamental understanding and realistic theoretical description of martensitic phase transformations and the influence of different defects on the kinetics require different length and time scales and demand atomistic resolution of the approach

*Corresponding author: a.v.karavayev@vniitf.ru 
used. So, large-scale Classical Molecular Dynamics (CMD) is utilized to simulate the process of the $b c c \rightarrow h c p$ phase transition. CMD simulations are performed using the LAMMPS code [2] with the Embedded Atom Model (EAM) interatomic potential for pure $\mathrm{Ti}$ [3] which reasonably well describes many properties of $h c p, b c c$ and liquid Ti including the equilibrium phase diagram at relatively low pressures (see Table 1). We employ the OVITO [4] software to visualize the resulted atomic configurations and the Polyhedral Template Matching method (PTM) [5] to distinguish between different structure types. Such settings allow us to perform simulations and postprocessing of sufficiently large systems containing hundreds millions of atoms.

Table 1. Thermodynamically equilibrium parameters of the $\alpha-\beta$ phase transition and melting at zero pressure for pure Ti calculated within CMD using the Thermodynamic Integration Method [6,7] for the EAM interatomic potential [3] in comparison with experimental data $[8,9]$.

\begin{tabular}{|c|c|c|c|c|c|c|}
\hline Property & $T_{\alpha \beta}, \mathrm{K}$ & $\begin{array}{c}\Delta H_{\alpha \beta}, \\
\mathrm{eV} / \text { atom }\end{array}$ & $\begin{array}{c}\Delta V_{\alpha \beta}, \\
\AA^{3} / \text { atom }\end{array}$ & $T_{m}, \mathrm{~K}$ & $\begin{array}{c}\Delta H_{m}, \\
\mathrm{eV} / \text { atom }\end{array}$ & $\begin{array}{c}\Delta V_{m}, \\
\AA^{3} / \text { atom }\end{array}$ \\
\hline Experiment & 1155 & 0.0435 & -0.154 & 1941 & 0.157 & 0.69 \\
\hline CMD, EAM & 1120 & 0.022 & -0.089 & 1920 & 0.128 & 0.408 \\
\hline
\end{tabular}

\section{Polycrystalline CMD sample generation}

Large-scale CMD simulations are performed within an elongated rectangular simulation box with a size of approximately $80 \times 655 \times 80 \mathrm{~nm}$ that corresponds to $\sim 250$ million atoms. Polycrystalline samples with the $b c c$ lattice are prepared through crystallization from a liquid state and subsequent recrystallization at elevated temperatures controlling the resulting polycrystalline structures by the use of various heat treatment regimes (see [10]).

At the initial moment the system is set as $b c c$ single crystal. After gradual heating at constant zero pressure up to $2200 \mathrm{~K}$ the system melts down. Then during subsequent gradual cooling well below the melting temperature $\sim 1200 \mathrm{~K}$ the bulk crystallization to $b c c$ lattice occurs and the polycrystalline structures of samples are formed. The variation of the cooling-heating regimes allows us to control the recrystallization rate and resulting grain structures. In such a way we prepared two kinds of samples. The first one is obtained by the subsequent annealing of the crystalized system at $1900 \mathrm{~K}$ during $4 \mathrm{~ns}$. It is a system where almost all grain boundaries are normal to the $y$-axis (see Fig. 1), that is, the obtained disoriented grains have effective infinite length along the $x$ and $y$ axes due to periodic boundary conditions used in the Cartesian directions. The average grain size along the $y$ axis is $\sim 82 \mathrm{~nm}$. This bamboo-like structure looks as a reasonable model of a real material with a grain size bigger than the size-scale available for state-of-the-art large-scale CMD. The second kind of samples under consideration is obtained by annealing at lower temperature $1400 \mathrm{~K}$ during $0.8 \mathrm{~ns}$. Such a heat treatment anneals only very fine grains and results in a fine nanocrystalline $b c c$ structure with $\sim 30 \mathrm{~nm}$ average grain size (see Fig. 2). The employed heating-cooling procedure performed in the NPT-ensemble gives many benefits. The main advantages are realistic grain boundaries between mutually disoriented crystal grains produced during solidification and subsequent recrystallization and the presence of "natural" ingrain defects. For example, in Fig. 1 one can see edge dislocations with $1 / 2$ [111] Burgers vector, dislocation loops and numerous point defects. The samples were relaxed at zero pressure and $T=600 \mathrm{~K}$, which is noticeably lower than the equilibrium $\beta-\alpha$ transition temperature (see Table 1), i.e., the bcc phase is metastable at these conditions. Nevertheless, the spontaneous $\beta-\alpha$ transformation at $600 \mathrm{~K}$ and zero pressure doesn't occur in the tens nanosecond time-scale in the samples of both kinds. 

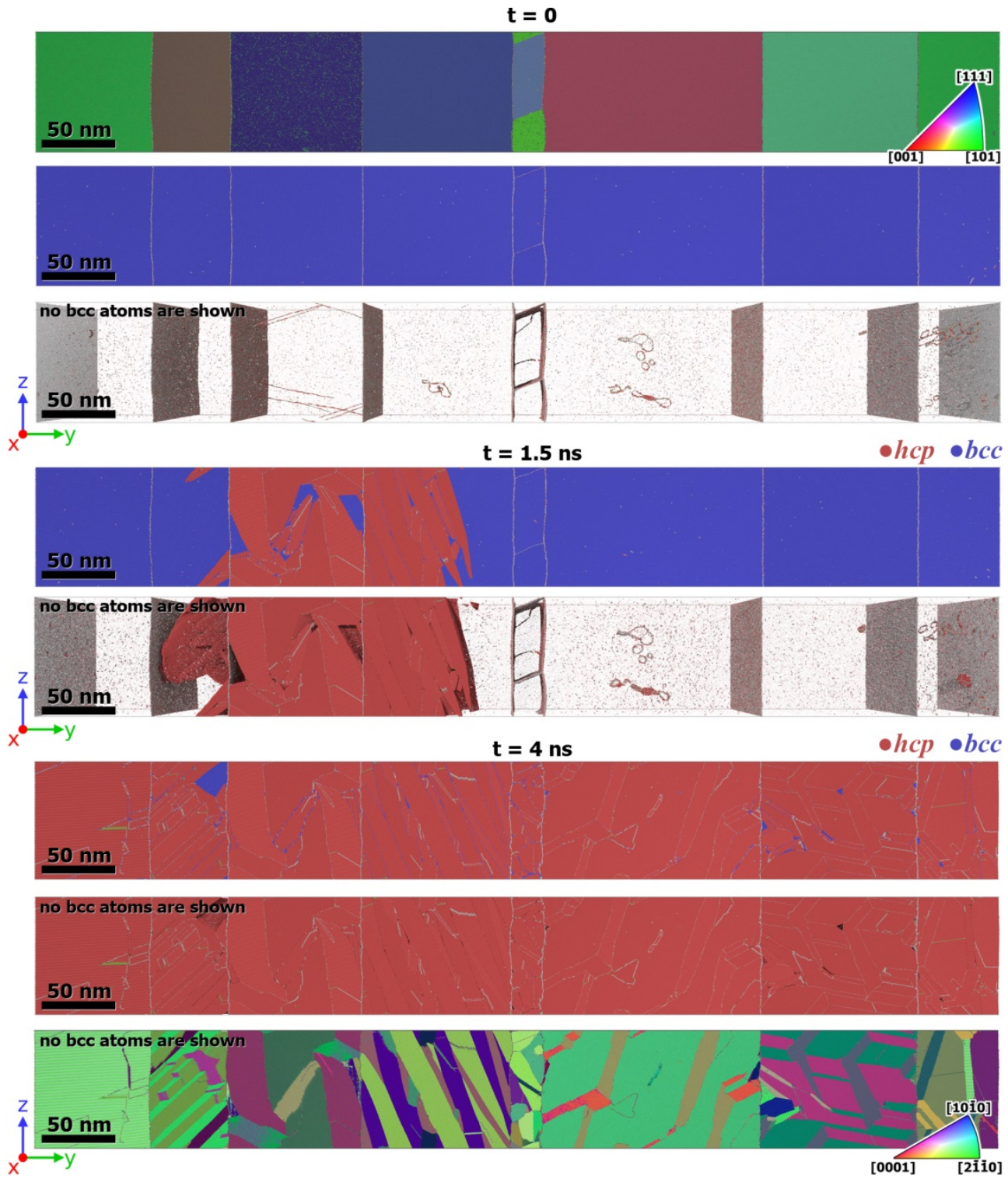

Fig. 1. Destruction of the metastable $\beta$ phase $(\beta \rightarrow \alpha$ martensitic transition) in a bamboo-like coarse grain sample during cooling from $T=600 \mathrm{~K}$ down to room temperature.

\section{$3 \beta \rightarrow \alpha$ transition caused by external influences}

With the prepared metastable samples of two kinds with coarse and fine bcc grain structures we performed the CMD study of the $\beta \rightarrow \alpha$ phase transition under further cooling down to room temperature, relatively weak plain shock wave pressures and imposed shear deformations. During the cooling of the samples in the NPT-ensemble down to room temperature the transition to the $\alpha$ phase starts without another external influence in both the bamboo-like and fine grain samples at approximately the same temperature of $\sim 475 \mathrm{~K}$ for the transition onset (see Figs. 1-3). In the coarse grain sample the dislocations act as the source of the $b c c$ to $h c p$ transition whereas the dislocation loops "lock" the newly growing 
$\alpha$ phase inside themselves. In the case of the fine grain sample there are two sources of the newly formed $h c p$ lattice appearing approximately at the same time: the grain boundary in the central part and the free surface at the right end of the sample. As one can see in Figs. 2 and $3 \mathrm{~b}$, the simulation time of $12 \mathrm{~ns}$ is insufficient to complete the $b c c \rightarrow h c p$ phase transition. It requires much longer times to transform the retained $\beta$ phase pent-up by numerous grain boundaries.
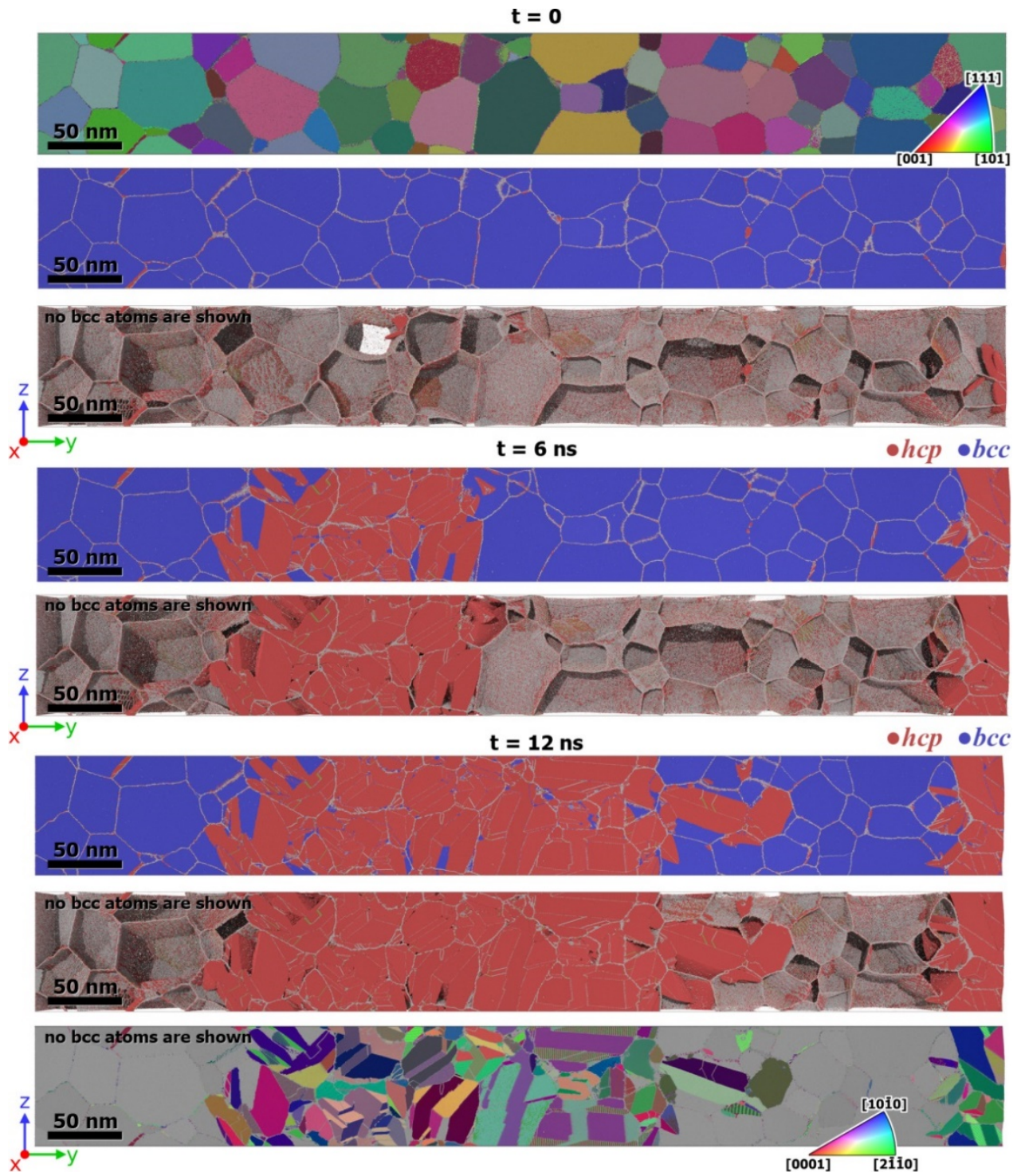

Fig. 2. Destruction of the metastable $\beta$ phase ( $\beta \rightarrow \alpha$ martensitic transition) in the fine grain sample during cooling from $T=600 \mathrm{~K}$ down to room temperature.

In both cases the transition happens as martensitic structure rearrangement with a typical needle-like pattern and numerous twins. During the transition at the $\alpha-\beta$ crystal interfaces the lattice orientations obey the well-known Burgers orientation relationship [11]: $(1 \overline{1} 0)_{\beta} / /(0001)_{\alpha},[111]_{\beta} / /[11 \overline{2} 0]_{\alpha}$. Figure $3 \mathrm{c}$ shows the terraced $\alpha-\beta$ crystal interface. The bent of the direction from $[111]_{\beta}$ to $[11 \overline{2} 0]_{\alpha}$ at the $\alpha-\beta$ interface indicates 
that additional rigid-body rotation to a small angle is required to realize the Burgers orientation relationship. Thus, there are local internal shear stresses caused by the transitions in the samples. This is consistent with both theoretical [12] and experimental [13] works. The final $\alpha$ structures inherit the prior $\beta$ grain boundaries. The results of the simulations show that the initial $b c c$ grain boundaries prohibit the $h c p$ lattice penetration and growth. This leads to significantly slower $\beta \rightarrow \alpha$ transition kinetics in the fine grain sample (see Fig. 3b).

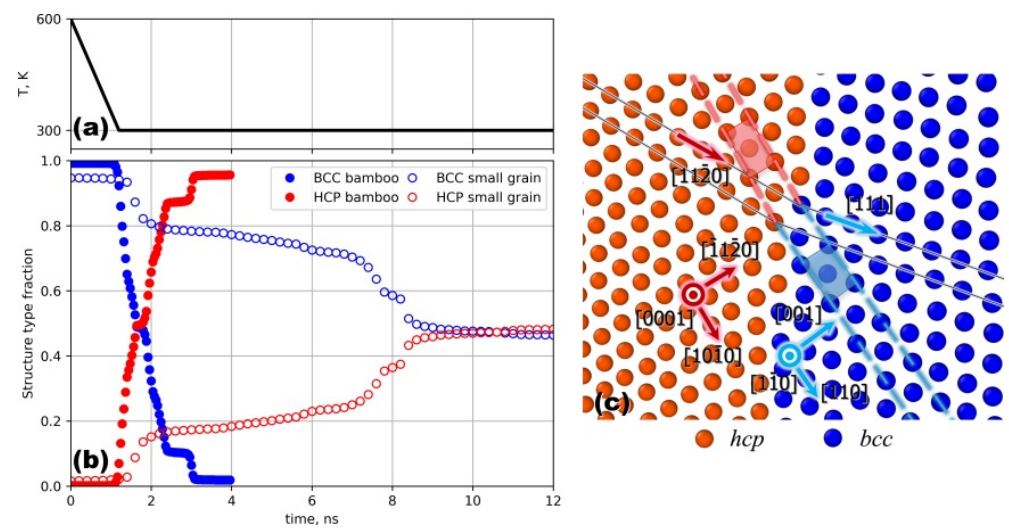

Fig. 3. Kinetics and mechanism of the martensitic $\beta \rightarrow \alpha$ phase transitions in bamboo-like coarse grain and fine grain samples. (a) - temperature of the thermostat during cooling from $T=600 \mathrm{~K}$ down to room temperature; (b) - fraction of atoms of $b c c$ and $h c p$ structures in the samples; (c) - mutual orientation of prior $\beta$ and daughter $\alpha$ phases during the martensitic $\beta \rightarrow \alpha$ phase transition.

Another type of external influence under consideration is the dynamic loading of metastable $b c c$ samples by relatively weak plain shock waves propagating in the elongated direction of the samples. The shock waves are generated by imparting the oncoming initial velocities to the left and right halves of the samples. In this study we consider shock wave amplitudes in the range 1-3 GPa which are not sufficient to trigger the transition to the $\omega$ phase. According to results of our CMD simulations, the $h c p$ lattice nucleation at dislocations and dislocation loops which are now mobile under stresses behind the shock front is preferable, as shown in Fig. 4 on the left. Here one can see the expanding loop favoring for the transition development. Other sources of the newly growing $h c p$ phase are some grain boundaries and free surfaces of the samples. In all cases the $\beta \rightarrow \alpha$ phase transition does not start instantaneously after shock front passing, but it takes some time (up to $0.3 \mathrm{~ns}$ depending on the shock wave magnitude and sample kind) to develop the $\alpha$ phase nuclei in the following release wave. Weaker waves and finer grain structures require longer incubation times. As the $h c p$ nuclei form, the subsequent transformation occurs as thin $\alpha$ phase lamellae growth with the characteristic velocity order of shear sound speed in the bcc lattice. This growth is accompanied by a slow expansion of the lamellae in the transverse direction (see Fig. 4).
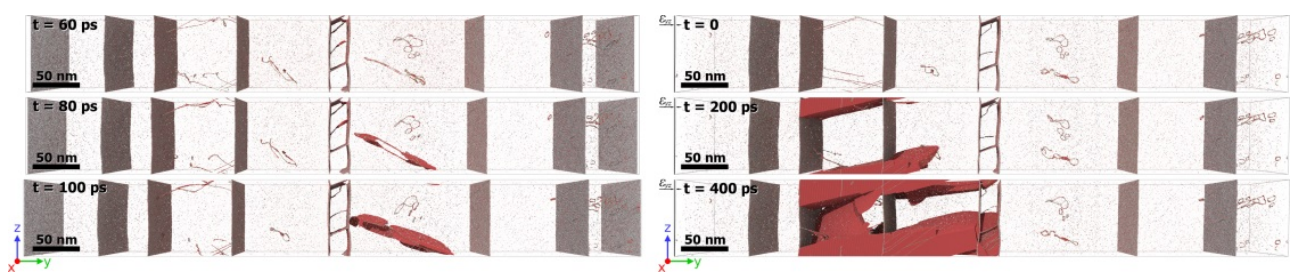

Fig. 4. Initial stages of metastable $\beta$ phase destruction in a bamboo-like coarse grain sample after the passage of a plain shock wave of $\sim 3 \mathrm{GPa}$ and the following release wave in the sample (on the left) and under imposed shear deformation $\varepsilon_{y z}=0.02$ (on the right). No $b c c$ atoms are shown. 
The last case in the focus of our study is small shear deformations in the range of $\varepsilon_{i j}=0.005-0.02$ that we instantly impose on the metastable $\beta$ polycrystalline samples of both kinds in different directions. For all shear deformations, the $\beta \rightarrow \alpha$ phase transition takes noticeable incubation times to develop the $h c p$ nuclei. Again in the coarse grain sample the main source of the newly growing $\alpha$ phase is ingrain extended defects - edge dislocations and dislocation loops. While in the fine grain sample the $h c p$ nuclei are produced by some grain boundaries. In all the cases of the plain shock wave and shear deformation grain boundaries hamper the hcp phase penetration and growth.

\section{Summary}

Large-scale ( $\sim 250$ million atoms) CMD is utilized to study metastable $\beta$ phase destruction in pure Ti. CMD samples with fine and coarse metastable polycrystalline $b c c$ structures are prepared through crystallization from a liquid state and subsequent recrystallization at elevated temperatures. The metastable $b c c$ samples were relaxed at temperatures noticeably lower than the equilibrium $\beta-\alpha$ transition temperature. To trigger the $\beta \rightarrow \alpha$ martensitic phase transition we use three kinds of external disturbance - further cooling down to room temperature, relatively weak plain shock waves and imposed shear deformations. Massively-parallel large scale CMD simulations with the use of computationally efficient yet accurate interatomic interaction models like EAM give an opportunity to obtain qualitative and quantitative information about solid-solid phase transformation barriers, mechanisms, and kinetics in direct atomistic simulations for representative virtual CMD samples. Originally it was supposed that disordered grain boundaries would serve as triggers of the transition and the finer grain structure would show faster transition. But results of CMD simulations evidence that grain boundaries despite their role as transition origins hamper the hcp phase penetration into neighbor grains and its following growth. In the cases of weak shock waves and shear deformations, appearing shear stresses lead to mobility of dislocations that serves as a favoring factor for the transition initiation and development. Thus, the dislocation loop which locks the hcp phase inside in the case of cooling proved to be a conductor of the transition development in the particular grain.

\section{References}

1. J. Zhang et al., J. Phys. Chem. 69, 2559 (2008)

2. S. Plimton, J. Comp. Phys. 117, 1 (1995)

3. M.I. Mendelev, T.L. Underwood, G.J. Ackland, J. Chem. Phys. 145, 154102 (2016)

4. A. Stukowski, Model. Simul. Mater. Sci. Eng. 18, 015012 (2010)

5. P.M. Larsen, S. Schmidt, J. Schiotz, Model. Simul. Mater. Sci. Eng. 24, 055007 (2016)

6. D. Frenkel, A.J.C. Ladd, J. Chem. Phys. 81, 3188 (1984)

7. R. Freitas, M. Asta, M. de Konig, Comp. Mat. Sci. 112, 333 (2016)

8. E.Y. Tonkov, E.G. Ponyatovsky, Phase transformations of elements under high pressure (CRC Press, Boca Raton, 2000)

9. K.C. Mills, Recommended values of thermophysical properties for selected commercial alloys (Woodhead Publishing Limited, Cambridge, 2002)

10. V.V. Dremov, P.V. Chirkov, and A.V. Karavaev, Sci. Rep. 11, 934 (2021)

11. W.G. Burgers, Physica I, 561 (1934)

12. M. Kato, S Onaka, T. Fujii, Sci. Tech. Adv. Mat. 2, 375 (2001)

13. D. He et al., Mat. Sci. Eng. A 549, 20 (2012) 